\title{
Structure and Stability of Filamentary Clouds Supported by Lateral Magnetic Field
}

\author{
Tomoyuki Hanawa ${ }^{1}$ and Kohji Tomisakar ${ }^{2}$ \\ ${ }^{1}$ Center for Frontier Science, Chiba University, Chiba, 263-8522, Japan \\ email: hanawa@faculty.chiba-u.jp \\ ${ }^{2}$ Division of Theoretical Astronomy, National Astronomical Observatory of Japan, Mitaka, \\ Tokyo 181-8588, Japan \\ email: tomisaka@th.nao.ac.jp
}

\begin{abstract}
We have constructed two types of analytical models for an isothermal filamentary cloud supported mainly by magnetic tension. The first one describes an isolated cloud while the second considers filamentary clouds spaced periodically. The filamentary clouds are assumed to be highly flattened in both the models. The former is proved to be the asymptotic limit of the latter in which each filamentary cloud is much thinner than the distance to the neighboring filaments. These models show that the mass to flux ratio is crucial for the magnetohydrodynamical equilibrium. The upper bound for the line density, i.e., the mass per unit length, is proportional to the magnetic flux. The mass to flux ratio is slightly larger than the critical value, $(2 \pi \sqrt{G})^{-1}$, in the first model and lower in the second model. The first model is unstable against fragmentation and the wavelength of the fastest growing mode is several times longer than the cloud diameter. The second model is likely to be unstable only when the mass to flux ratio is supercritical.
\end{abstract}

Keywords. clouds, magnetic field, stability

\section{Introduction}

Molecular clouds are often filamentary in shape and threaded by an ordered magnetic field. The magnetic field is expected to play a significant role in the dynamics of the filamentary clouds. The magnetic force, $\boldsymbol{j} \times \boldsymbol{B}$, is perpendicular to the magnetic field. Thus the direction of the magnetic field is crucial to the cloud dynamics. Although the structure and dynamics of magnetized filamentary clouds have been studied extensively, most of them deal with magnetic fields running either parallel to or spiraling around the cloud axis. As far as we know, only Tomisaka (2014) has studied the structure of filamentary clouds threaded by magnetic field perpendicular to the cloud axis.

A filamentary cloud is highly flattened when it is threaded by a strong magnetic field perpendicular to the axis as shown in Tomisaka (2014). Then we can apply the thin disk approximation for analyzing the cloud structure, since the cloud thickness parallel to the mean magnetic field is much smaller than the cloud width. Using the thin disk approximation, Hanawa \& Tomisaka (2015) have obtained analytical solutions for the structure of filamentary clouds threaded by magnetic field perpendicular to the cloud axis. This paper summarizes the main results of Hanawa \& Tomisaka (2015).

\section{Results}

We assume that filamentary clouds are highly flattened and gas is confined in a lazar thin sheet. Then the magnetic field should be force free outside the sheet. In other words, the electric current is also confined inside the sheet and the magnetic field is bent on the plane of the sheet. Then we can evaluate the self-gravity and magnetic field by applying the method of mirror image. A simple analytic solution is found for the magnetohydrodynamic equilibrium in which 
the self-gravity is balanced by the sum of the magnetic tension and isothermal gas pressure. The analytic solution has the density $(\rho)$ and magnetic field $(\boldsymbol{B})$ distribution,

$$
\begin{aligned}
\rho(x, y, z) & =\frac{\lambda}{\pi} \frac{a \delta(x)}{\pi a^{2}+x^{2}}, \quad \boldsymbol{B}=\left(\begin{array}{c}
B_{x} \\
B_{z}
\end{array}\right)=-\frac{\Phi}{\pi\left[x^{2}+(|z|+a)^{2}\right]}\left(\begin{array}{c}
\frac{z x}{|z|} \\
|z|+a
\end{array}\right), \\
\lambda & =\frac{c_{s}^{2}}{2 G}+\sqrt{\left(\frac{\Phi}{2 \pi \sqrt{G}}\right)^{2}+\left(\frac{c_{s}^{2}}{2 G}\right)^{2}},
\end{aligned}
$$

in the Cartesian coordinate, $(x, y, z)$, where the symbols, $a, c_{s}, G, \lambda$, and $\Phi$ denote the cloud width, the isothermal sound speed, the the gas mass per unit length, the gravitational constant, and the magnetic flux per unit length, respectively. The mass to flux ratio is constant at $\lambda / \Phi$ and supercritical in this solution. This solution is proved to be unstable against fragmentation by using the variational principle. The fastest growing mode has the wavelength several times longer than the cloud diameter.

In the second solution filamentary clouds are arranged periodically on a plane. The density and magnetic field distribution are expressed as

$$
\rho(x, y, z)=\frac{\lambda \sinh w}{\ell[\cosh w-\cos (2 \pi x / \ell)]}, \quad B_{z}(x, y, z=0)=\frac{1}{\ell} \sum_{j=0}^{\infty} b_{j} \cos \left(\frac{2 \pi x}{\ell}\right),
$$

where $\ell$ and $w$ specify the cloud separation and cloud width, respectively. The magnetic field is expressed by the Fourier series. Only when

$$
b_{0} \geqslant 2 \pi \sqrt{G} \sqrt{\lambda\left(\lambda-\frac{c_{s}^{2}}{G}\right)},
$$

we can find an equilibrium solution. When the equality holds, the magnetic field is expressed as

$$
\rho(x, y, z)=\frac{b_{0} \sinh w}{\ell[\cosh w-\cos (2 \pi x / \ell)]} .
$$

This critical solution reduces to Eq. (2.1) in the limit of $w \rightarrow 0$ while $w \ell=a$. Thus this critical solution should be unstable against fragmentation. When $b_{0}$ is larger than the critical value, the filamentary cloud is surrounded by nearly uniform magnetic field. When $w \gg 1$, Eq. (2.3) describes a uniform slab. Nakano \& Nakamura (1978) proved that such a slab is unstable against fragmentation only when it is supercritical, i.e., $b_{0}>2 \pi \sqrt{G} \lambda$ in our model. We think that our second solution is likely to be unstable against fragmentation only when the mass to flux ratio is supercritical. See Hanawa \& Tomisaka (2015) for further details.

\section{References}

Hanawa, T., Tomisaka K. 2015, ApJ, 801, 11

Nakano, T., Nakamura, T. 1978, PASJ, 30, 671

Tomisaka, K. 2014, ApJ, 785, 24 